Results 100,801 registered individuals made 2.5 million trips between July 2010 and March 2011. Compared with local residents and workers, registered individuals were more likely to be male and to live in areas of low deprivation and high cycling prevalence. Among those registered, females made 1.63 (95\%CI 1.74,1.53) fewer trips per month than males. In combination with the fact that fewer females registered in the first place, this meant that only $17.8 \%$ of the total number of $\mathrm{BCH}$ trips were made by females. Adjusting for the fact that deprived areas were less likely to be close to $\mathrm{BCH}$ docking stations, users in the most deprived areas made $0.85(95 \%$ CI $0.63,1.07)$ more trips per month than those in the least deprived areas.

Conclusion Females and residents in deprived areas are underrepresented among users of London's public bicycle sharing scheme. Indeed, the $\mathrm{BCH}$ scheme currently appears to be less gender-equal than cycling in general in London. Nevertheless, registered users in more deprived areas made more trips on average, suggesting there may be a greater latent demand for cycling in these areas. The scheme's expansion into more deprived areas from Spring 2012 has, therefore, the potential to create a more socio-economically equitable uptake of cycling.

\section{OP35 THE HEALTH IMPACTS OF FREE BUS TRAVEL FOR YOUNG PEOPLE IN LONDON}

doi:10.1136/jech-2012-201753.035

${ }^{P}$ Edwards, ${ }^{2 R}$ Steinbach, ${ }^{2 P}$ Wilkinson, ${ }^{2} \mathrm{M}$ Petticrew, ${ }^{1} \mathrm{~A}$ Goodman, ${ }^{2} \mathrm{~A}$ Jones, ${ }^{3 \mathrm{H}}$ Roberts, ${ }^{4} \mathrm{C}$ Kelly, ${ }^{4} \mathrm{~J}$ Nellthorp, ${ }^{2} \mathrm{~J}$ Green. ${ }^{1}$ Faculty of Epidemiology and Population Health, LSHTM, London, UK; ${ }^{2}$ Faculty of Public Health and Policy, LSHTM, London, UK; ${ }^{3}$ nnstitute of Child Health, UCL, London, UK; "4nstitute for Transport Studies, University of Leeds, Leeds, UK

Background Interventions in transport systems have potentially far-reaching impacts on public health, but can be challenging to evaluate. In 2005, young people in London gained access to free bus travel; an intervention that has a number of potential risks and benefits to health. As transport access is linked to well-being, we might expect the policy to benefit the health of young people by reducing transport exclusion. However, health effects might also include: young people doing less walking, thus reducing levels of physical activity, but also reducing exposure to pedestrian injury risk; or being more exposed to assault as they travel further.

Methods We utilized change-on-change analyses comparing pre (2001-2004) and post (2006-2009) changes in outcomes in 'younger people' (intervention group, 12-17 years) to 'adults' (control group, 25-59 years) in London, UK. Main outcome measures included changes in travel patterns (trips made by main travel mode and distances travelled), road traffic injuries and hospital admissions for assault.

Results Post-intervention, the total number of journeys to school or work made by younger people increased relative to adults (change-on-change ratio 1.19: 95\% CI 1.13-1.25), and the proportion of short trips $(<1 \mathrm{~km})$ by bus doubled (1.97: 1.07-3.84). There was some evidence that younger people made fewer trips where walking was the main mode of travel (0.76: 0.70-0.85), but no evidence for a change in overall distances walked by younger people post-intervention. Against background declines in road traffic injury, the decrease in road injury to young people was larger relative to adults $(0.84 ; 0.82-0.87)$, however pedestrian injuries declined similarly in both groups. Rates of hospitalisation due to assaults increased in younger people relative to adults (1.20: 1.13-1.27).

Conclusion A change in the distribution of travel modes used by younger people (relative to adults who had not received free bus travel) was observed post-intervention. Younger people made fewer trips where walking was their main mode of travel, but there was little overall difference in distances walked, suggesting that the policy may have generated journeys but made little overall impact on prevalence of active transport. Observed changes in road traffic injuries reflect the relative risks of changing travel modes. The intervention has been associated with a small relative increase in assaults to younger people. A change-on-change analysis has enabled us to use this 'natural experiment' to quantify some important health outcomes of a transport policy in the absence of evidence from a randomised trial.

\section{OP36 A CROSS-SECTIONAL ASSESSMENT OF THE EFFECT OF THE FREE OLDER PERSONS' BUS PASS ON ACTIVE TRAVEL AND REGULAR WALKING AMONG ADULTS $\geq 60$ YEARS IN ENGLAND USING DATA FROM THE NATIONAL TRAVEL SURVEY 2005-2008}

doi:10.1136/jech-2012-201753.036

${ }^{1} \mathrm{~S}$ Coronini-Cronberg, ${ }^{1,2} \mathrm{C}$ Millett, ${ }^{1} \mathrm{~A}$ Laverty, ${ }^{1,3}{ }^{2} \mathrm{E}$ Webb. ${ }^{1}$ Department of Primary Care and Public Health, Imperial College London, London, UK; ${ }^{2}$ Clinical Programme Group 7 , Imperial College NHS Trust, London, UK; ${ }^{2}$ ESRC International Centre for Lifecourse Studies in Society and Health, UCL, London, UK

Background The benefits of physical activity for all age groups is well-documented and there is increasing interest in the promotion of incidental physical activity, such as active transport, which includes walking, cycling and use of public transport. For older adults, even small increases in activity may have significant benefits: for example, the relative risk of disability is reduced by $7 \%$ for each additional hour of relatively gentle physical activity undertaken each week. Our study assessed the potential public health benefit of the National Bus Pass, introduced in 2006, which permits free local bus travel for older adults ( $\geq 60$ years) in England.

Methods Data from the year prior to the pass being introduced (2005) to the most recently available (2008) were extracted from an annual cross-sectional survey, the National Transport Survey, resulting in a sample size of 15175 older adults. Models assessed associations between possessing a bus pass and our main outcome measures: use of active transport (walking, cycling and use of public transport), use of buses and walking three or more times a week. Since participants were sampled by household, all models were adjusted for clustering at the household level, as well as a range of confounders, including: age, sex, and socio-economic status.

Results Preliminary results show that having a free pass is significantly associated with greater use of active travel among both disadvantaged and advantaged groups. It is also associated with increased use of buses and a greater likelihood of walking three or more times a week.

Conclusion Older people in England with a free bus pass are more likely to use active transport, buses and undertake regular walking than those without, regardless of their socio-economic status. This suggests public subsidies enabling free bus travel for older persons may confer significant population health benefits through increasing incidental physical activity levels.

\section{Prevention}

\section{OP37 PSYCHOLOGICAL CONSEQUENCES OF FALSE-POSITIVE SCREENING MAMIMOGRAMS IN THE UK: A SYSTEMATIC REVIEW}

doi:10.1136/jech-2012-201753.037

${ }^{1} \mathrm{M}$ Bond, 'T Pavey, ${ }^{2} \mathrm{~K}$ Welch, 'C Cooper, ${ }^{1} \mathrm{R}$ Garside, 'S Dean, ' $\mathrm{C}$ Hyde. 'Peninsula Medical School, University of Exeter, Exeter, UK; ${ }^{2}$ Karen Welch Information Consultancy, Fareham, UK

Background In the UK women aged 47-73 are invited for screening by mammography every three years. In 2009-10 more than 2.24 million women in this age group in England were invited to take 
part in the programme, of these $73 \%$ attended a screening clinic. Of these 64,104 were recalled for assessment. Of those recalled $81 \%$ did not have breast cancer, these women had a false-positive mammogram. The aim of this systematic review was to identify the psychological impact of false-positive screening mammograms and evidence for the effectiveness of interventions designed to reduce this.

Methods This was a systematic review using the principles of the Centre for Reviews and Dissemination in York.

All UK controlled studies and qualitative studies of women with a false-positive screening mammogram were included. The control group participants had normal mammograms. All psychological outcomes including returning for routine screening were permitted. Titles and abstracts were screened independently by two reviewers. Retrieved papers were reviewed and selected using the same independent process. Data were extracted by one reviewer and checked by another. Each included study was assessed for risk of bias.

Results The searches returned seven includable studies $(7 / 4,423)$. Those using disease specific measures found that there could be enduring distress that lasted up to three years; the level of distress was related to the degree of invasiveness of the assessment. At three years the relative risks were, further mammography 1.28 (95\% confidence interval 0.82 to 2.00), fine needle aspiration 1.80 (95\% confidence interval 1.17 to 2.77 ), biopsy 2.07 ( $95 \%$ confidence interval 1.22 to 3.52). There was also an enduring impact for those put on early recall 1.82 (95\% confidence interval 1.22 to 2.72), However, studies that used generic measures of anxiety and depression found no such impact up to three months after screening. The strongest evidence suggests that women with false-positive mammograms have an increased likelihood of failing to reattend for routine screening, relative risk 0.97 ( $95 \%$ confidence interval 0.96 to 0.98 ) compared to women with normal mammograms. No studies of women from different socio-economic or ethnic groups were found nor any published qualitative studies.

Conclusion Having a false-positive screening mammogram can cause breast cancer specific distress for up to three years. It is less likely there will be general anxiety or depression detectable at pathological levels. The degree of distress is related to the invasiveness of the assessment. Women with false-positive mammograms are less likely to return for routine assessment than those with normal ones.

\section{OP38 ACCOUNTING FOR NON-UPTAKE OF BOWEL CANCER SCREENING: A QUALITATIVE STUDY}

doi:10.1136/jech-2012-201753.038

C Palmer, M Thomas, R Raine, C Von Wagner. Department of Epidemiology and Public Health, UCL, London, England

Background Since being established in 2006, the English NHS Bowel Cancer Screening Programme (NHS BCSP) offers the faecal occult blood (FOB) test to adults aged $60-74$. This bowel cancer screening test is sent by post every two years to recipients who are required to provide six small samples of faeces from three bowel movements. The kit is returned by post to be tested for minute traces of blood that may be an early warning sign for the development of bowel cancer. Analysis shows that approximately $53 \%$ of people offered bowel cancer screening by FOB test take it up, and that uptake varies from $35 \%$ to $60 \%$ in the most and least deprived areas, respectively.

Methods We used a qualitative approach to explore views and experiences about bowel cancer screening in people who did not take part in the screening programme when invited to do so. We conducted 16 focus groups in London and South Yorkshire to generate extensive textual data, and analysed our data thematically using grounded theory techniques to explore and examine accounts of non-uptake. Previous research examining 'non-uptake', of this and other medical interventions has identified considerable challenges in terms of identifying and recruiting individuals who may be defined in this way. We therefore used a novel recruitment approach, working with the National BCSP Hubs to identify and recruit 'nonuptakers' of bowel cancer screening. We present the findings from our thematic analysis of 16 focus groups undertaken with people who did not participate in bowel cancer screening and explore the complexity of "non-uptakers" accounts.

Results \& Conclusion During preliminary analyses, we identified common accounts through which participants rationalised nonuptake and which present one or several barriers to undertaking bowel cancer screening. Participants rationalised their non-uptake of screening in a number of ways, for example by making reference to perceptions of low personal risk informed by 'feeling well' or having had no family history of bowel cancer. Others expressed sophisticated doubts about the benefits or value of the FOB test specifically, or in relation to diagnostic or treatment interventions for cancer more generally. Finally, the necessity to provide samples of faeces to complete the FOB test emerged repeatedly as a troubling aspect of the bowel cancer screening methodology offered by the English NHS BCSP.

\section{OP39 PROTECTION BY BCG AGAINST TUBERCULOSIS: SYSTEMATIC REVIEW AND META-REGRESSION ANALYSIS}

doi:10.1136/jech-2012-201753.039

'P Mangtani, ' $\mathrm{C}$ Ariti, ${ }^{1} \mathrm{~L}$ Rodrigues, ${ }^{2} \mathrm{~A}$ Abubakar, ${ }^{3} \mathrm{~L}$ Pimpin, ${ }^{1} \mathrm{P}$ Fine, ${ }^{1} \mathrm{P}$ Smith, ${ }^{4} \mathrm{~J}$ Sterne. ${ }^{1}$ Epidemiology and Population Health, LSHTM, London, UK; '2Department of Medicine, University of East Anglia, Norwich, UK; ${ }^{3}$ Department of Epidemiology, University of Cambridge, Cambridge, UK; ${ }^{4}$ Department of Epidemiology , University of Bristol, Bristol, UK

Setting Reasons for variation in BCG protection against tuberculosis are not well understood. We therefore estimated the protection provided by BCG vaccination against tuberculosis and examined reasons for variation in different settings.

Methods We systematically searched English and non-English articles in 10 databases from inception to May 2009 as well as sources such as Google Scholar and trial registers to October 2009. Search terms included tuberculosis, tubercle bacill*, M. tuberculosis, $M$. bovis, $M$. africanum, $M$. canetti, $M$. microti and $M$. tuberculosis. Terms for the intervention included BCG Vaccine, BCG, BCG Vacc*, BCG Imm*, bacillus calmette.

Two reviewers independently screened studies for inclusion. Data (including results and domains of study quality) were extracted by one reviewer and checked by another. Pooled analyses were based on random and fixed effects meta-analysis. Heterogeneity was quantified using tau-squared $\left(\tau^{2}\right)$ statistics. Random-effects meta-regression was used to investigate associations of study characteristics with intervention effect estimates.

Results Of 21,030 references we identified 21 trials reporting tuberculosis outcomes. Protection against tuberculosis disease was variable $\left(\tau^{2}=0.292\right)$. BCG efficacy was high at higher latitude or when BCG was given only to infants $(62 \%, 95 \%$ CI $48-72 \%)$ or to children after strict screening for tuberculin sensitivity $72 \% 95 \% \mathrm{CI}$ 60-81\%) Protection against meningeal and miliary tuberculosis combined was higher in infants $(90 \%)$ and children with stringent testing respectively $(92 \%)$. The observed protective effect of BCG did not differ by the strain of BCG vaccine used. Stringency of tuberculin testing to exclude those infected or sensitised to mycobacteria explained a good proportion of the observed variation with latitude $\left(\tau^{2}=0.292\right.$ in the null model, 0.100 in the bivariate model). In a multivariable meta-regression analysis there was strong evidence that infant vaccination or stringent prior tuberculin testing before BCG in childhood was associated with increased efficacy $(p=0.02)$ after taking into account latitude and a measure of study quality-diagnostic detection bias. 